\title{
Oxymatrine ameliorates non-alcoholic fatty liver disease in rats through peroxisome proliferator-activated receptor- $\alpha$ activation
}

\author{
LIJUAN SHI ${ }^{1,2}$, LEI SHI ${ }^{3}$, HEFANG ZHANG ${ }^{1}$, ZHIJUAN HU $^{1,4}$, CHAO WANG $^{5}$, \\ DONGHUI ZHANG ${ }^{5}$ and GUANGYAO SONG ${ }^{1,6}$
}

\author{
${ }^{1}$ Department of Internal Medicine, Hebei Medical University, Shijiazhuang 050017; ${ }^{2}$ Department of Endocrinology, \\ The Third Hospital of Shijiazhuang City Affiliated to Hebei Medical University, Shijiazhuang 050011; \\ ${ }^{3}$ Department of Pharmaceutical Engineering, Hebei Chemical and Pharmaceutical Vocational Technology College, \\ Shijiazhuang 050026; Departments of ${ }^{4}$ Nephrology, ${ }^{5}$ Clinical Research Centre, and ${ }^{6}$ Endocrinology, \\ Hebei General Hospital, Shijiazhuang 050051, P.R. China
}

Received January 16, 2013; Accepted May 30, 2013

DOI: $10.3892 / \mathrm{mmr} .2013 .1512$

\begin{abstract}
Non-alcoholic fatty liver disease (NAFLD) is the most common type of liver disease worldwide. Recent studies have reported that oxymatrine (OMT), an active monomer isolated from Sophora flavescens Ait. (kushen), ameliorates NAFLD in rats. In order to explore the possible molecular mechanism involved, we used an NAFLD rat model with hyperlipidemia, which had been established by feeding a high-fructose diet (HFD) for eight weeks, and the model rats were subsequently treated with OMT $(80 \mathrm{mg} / \mathrm{kg} /$ day $)$ for a further four weeks. We evaluated the expression of genes and proteins regulating fatty acid oxidation and lipid export in the liver using quantitative (q)PCR and western blot analysis. The NAFLD model rats developed dyslipidaemia, hepatic steatosis and insulin resistance (IR). OMT administration for four weeks reduced body weight gain and visceral fat weight, decreased serum triglyceride (TG), total cholesterol (TC), free fatty acid (FFA) and fasting serum insulin (FinS) levels and lowered liver TG contents. It also increased the glucose infusion rate (GIR), indicative of a reduction in IR. Moreover, OMT treatment markedly increased the mRNA and protein levels of peroxisome proliferator-activated receptor- $\alpha$ (PPAR $\alpha$ ), carnitine palmitoyltransferase 1A (CPT1A) and microsomal triglyceride transfer protein (MTTP). The beneficial effects of OMT were further confirmed by the observation of a decrease
\end{abstract}

Correspondence to: Dr Guangyao Song, Department of Endocrinology, Hebei People's Hospital, 348 West Heping Road, Shijiazhuang, Hebei 050051, P.R. China

E-mail: sguangyao2@163.com

Abbreviations: NAFLD, non-alcoholic fatty liver disease; IR, insulin resistance; PPAR $\alpha$, peroxisome proliferator-activated receptor- $\alpha$; CPT1A, carnitine palmitoyltransferase 1A; MTTP, microsomal triglyceride transfer protein

Key words: oxymatrine, NAFLD, insulin resistance, lipid metabolism imbalance, PPAR $\alpha$, CPT1A, MTTP in lipid accumulation in the histology of the liver. Our results indicate that OMT may be used to treat NAFLD.

\section{Introduction}

Non-alcoholic fatty liver disease (NAFLD) is the most common liver disease worldwide, ranging from simple steatosis to cirrhosis and hepatocellular carcinoma (1). Hepatic steatosis [mainly triglycerides (TGs)] is believed to contribute to liver inflammation and nonalcoholic steatohepatitis progression (2). Therefore, it is important to identify factors that regulate hepatic lipid metabolism involved in the hepatic steatosis.

It has been reported that insulin resistance (IR) is a primary factor for the development of NAFLD (3). IR is associated with increased lipolysis and the reduced utilisation of free fatty acids (FFA) in adipose tissues, which leads to an elevated influx of FFA to the liver (4). Fatty acids are cytotoxic, and there are two metabolic methods for the influx of FFAs, one is by reesterification to TGs and the second is to participate in $\beta$-oxidation. These two methods are important in protecting the liver against the lipotoxicity of FFAs. Thus, if hepatic import and the synthesis of TGs exceeds hepatic TG oxidation and export, TGs accumulate in hepatocytes $(5,6)$ Therefore, a reduction in IR, the inhibition of FFA liberation, the promotion of FFA $\beta$-oxidation and export as very low density lipoproteins (VLDLs) may be effective strategies to decrease liver TG deposits.

A growing body of evidence indicates that peroxisome proliferator activated receptor- $\alpha$ (PPAR $\alpha)$ is important in the catabolism of fatty acids. PPAR $\alpha$ participates in numerous aspects of lipid metabolism, including fatty acid uptake through membranes, fatty acid binding in cells, fatty acid oxidation and lipoprotein assembly and transport (7), by regulating the expression of pivotal enzymes, such as carnitine palmitoyltransferase 1A (CPT1A) and microsomal triglyceride transfer protein (MTTP) (8). PPAR $\alpha$ agonists, such as fibrates, have been shown to be effective in the treatment of obesity and IR (9), and have been used to correct hypertriglyceridaemia and NAFLD in rats (10); thus, we used fenofibrate (FF) as a positive control. 
Oxymatrine (OMT) is one of the major components extracted from Sophora flavescens Ait. (kushen), a traditional Chinese medicine, and has been reported to possess various pharmacological effects, including anti-hepatitis virus infection, anti-hepatic fibrosis, anti-inflammation and anti-anaphylaxis activities and immune regulation (11-13). However, little is known about the effect of OMT on lipid metabolism, with the exception of a recent study, which reported that the oral administration of OMT for one week eliminated hepatic steatosis in fatty liver mice (14). New studies regarding matrine, another quinolizidine alkaloid isolated from Sophora flavescens Ait. (kushen) that has a chemical structure and pharmacological effects similar to those of OMT (Fig. 1), have demonstrated its beneficial effects on serum lipids and hepatic steatosis in vivo $(15,16)$. Additionally, matrine treatment decreases fat accumulation in 3T3-L1 preadipocytes in vitro (17). Based on the aforementioned studies, we hypothesise that OMT may have similar beneficial effects on the abnormal lipid metabolism in the high-fructose diet (HFD)-induced NAFLD rats.

The main aim of the present study was to investigate the effects of OMT on serum lipids, insulin sensitivity and hepatic steatosis in NAFLD rats. An additional goal was to explore whether these effects were associated with PPAR $\alpha$ activation.

\section{Materials and methods}

Drugs and reagents. OMT was obtained from Chia-Tai Tianqing Pharmacy Co., Ltd. (Jiangsu, China). FF (Lipanthyl; Laboratories Fournier, Diax, France) was purchased from The Third Hospital of Shijiazhuang City affiliated with Hebei Medical University (Shijiazhuang, China). The drugs were suspended in a $0.5 \%$ sodium carboxymethyl cellulose solution, respectively. Crystallised fructose was purchased from Hebei Huaxu Pharmaceutical Co., Ltd. (Shijiazhuang, China). Rabbit anti-rat PPAR $\alpha$ (ab8934) polyclonal antibody was purchased from Abcam Inc. (Cambridge, MA, USA). Rabbit anti-rat CPT1A (bs-2047R) and rabbit anti-rat MTTP (bs-5083R) polyclonal antibodies were purchased from Beijing Biosynthesis Biotechnology Co., Ltd. (Beijing, China). Liver tissue TG assays (E1013) and protein (BCA) assays (P1511) were obtained from Applygen Technologies Inc. (Beijing, China). Serum FFA diagnostic kits were purchased from Jiancheng Bioengineering Institute (Nanjing, China) and rat insulin radioimmunoassays were purchased from Millipore Corporation (Billerica, MA, USA). All other chemicals used in these experiments were purchased from Shanghai Sangon Biotechnology Co., Ltd. (Shanghai, China).

Animals. Thirty-six male Wistar rats aged 5-6 weeks (176-190 g) were provided by the Experimental Animal Center of Hebei Medical University (Shijiazhuang, China), housed individually in an air-conditioned room under controlled illumination (12-h light/dark cycle), temperature $\left(22-28^{\circ} \mathrm{C}\right)$ and humidity (40-60\%). The rats had free access to rodent chow and tap water throughout the experimental period and were allowed to acclimatise for 7 days prior to the experiment. The ethics committee of Hebei Medical University approved the experimental protocol and all animals received humane care in compliance with institutional animal care guidelines.

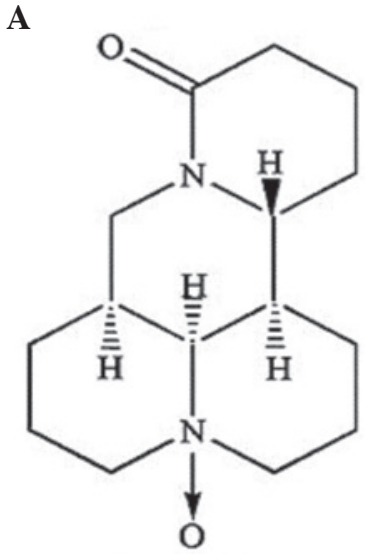

B

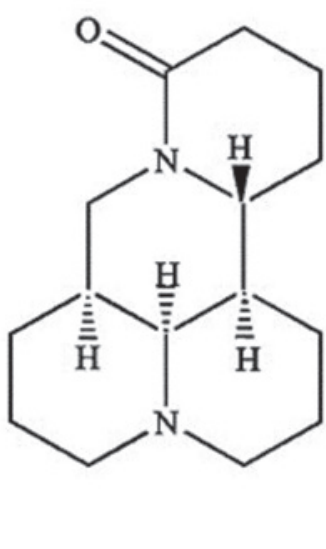

Figure 1. Chemical structure of (A) oxymatrine and (B) matrine.

Establishment of the NAFLD rat model and drug intervention. The rats were randomly separated into two groups, the standard diet (SD) group and the HFD group. The HFD (35\% fructose, $35 \%$ starch, $10 \%$ fat and $20 \%$ protein by energy) was prepared according to the literature $(18,19)$. At the end of the eighth week, four rats were selected from the HFD group and sacrificed, and their liver tissues were assessed for fatty liver development. Subsequently, the HFD rats were randomly subdivided into three groups $(\mathrm{n}=8)$ : the model (HFD), OMT $(80 \mathrm{mg} / \mathrm{kg} / \mathrm{day})$ and FF (30 mg/kg/day) groups. Drugs were administered intragastrically once a day for four weeks, while the SD and HFD groups were gavaged with an equivalent volume of the vehicle ( $0.5 \%$ sodium carboxymethyl cellulose solution). Food intake and body weights were monitored twice a week.

Following 4 weeks of treatment, rats were selected (three animals per group) for euglycemic hyperinsulinaemic clamping, then all rats were anaesthetised and sacrificed following an overnight fast. Blood samples were collected via abdominal aortic punctures and plasma was stored at $-20^{\circ} \mathrm{C}$. Visceral adipose tissue (VAT) from various anatomical locations (perirenal, epididymal and mesenteric) was dissected and weighed. The liver was quickly excised, frozen in liquid nitrogen and stored at $-80^{\circ} \mathrm{C}$ or fixed for further analysis.

Evaluation of insulin sensitivity with the euglycemic hyperinsulinaemic clamp. Rats were anaesthetised with pentobarbital $(35 \mathrm{mg} / \mathrm{kg}$, i.p.) and cannulas were implanted into the right jugular vein and the left carotid artery under aseptic conditions for infusion and sampling, respectively. Catheters were exteriorised at the back of the neck. Six days after cannulation surgery, a clamping test was performed as previously described (20) while rats achieved presurgery weight. The rats were continuously infused with regular human insulin (Novo Nordisk, Bagsvaerd, Denmark) at a rate of $10 \mathrm{mU} / \mathrm{kg} / \mathrm{min}$. During the insulin infusion, blood glucose was measured at $5 \mathrm{~min}$ intervals to maintain euglycaemia (at the fasting level) by adjusting the glucose infusion rate (GIR) using 30\% glucose. The average GIR $(\mathrm{mg} / \mathrm{kg} / \mathrm{min})$ during the final 30 min was used as an index of insulin sensitivity. Following testing, the animals were euthanised and the liver tissues were rapidly removed, freeze-clamped and stored at $-80^{\circ} \mathrm{C}$ for subsequent analysis. 
Table I. Primers sequences used for quantitative PCR.

\begin{tabular}{lll}
\hline Primer & \multicolumn{1}{c}{ Forward } & \multicolumn{1}{c}{ Reverse } \\
\hline PPAR $\alpha$ & 5'-GTACGGTGTGTATGAAGCCATCTT-3' & 5'-GCCGTACGCGATCAGCAT-3' \\
CPT1A & 5'-TGGTCAACAGCAACTACTACGC-3' & 5'-GAAGACGAATGGGTTTGAGTTC-3' \\
MTTP & 5'-CTTCTGCCTACACTGGCTACG-3' & 5'-GTTCTCCTCTCCCTCATCTGG-3' \\
GAPDH & 5'-TGAACGGGAAGCTCACTG-3' & 5'-GCTTCACCACCTTCTTGATG-3'
\end{tabular}

PPAR $\alpha$, peroxisome proliferator-activated receptor- $\alpha$; CPT1A, carnitine palmitoyltransferase 1A; MTTP, microsomal triglyceride transfer protein.

Biochemical analysis. Serum biochemistry, including serum TG, total cholesterol (TC) and alanine aminotransferase (ALT) levels were measured using standard laboratory procedures. Liver TGs were extracted from liver tissue homogenates and assessed using an assay kit and the values were normalised to the total protein concentration in the liver homogenate using a bicinchoninic acid (BCA) assay. Insulin levels were measured using a radioimmunoassay. Serum FFA levels were determined spectrophotometrically, according to the manufacturer's instructions.

Histology examination. Freshly isolated liver tissues were fixed in $10 \%$ formaldehyde PBS ( $\mathrm{pH} 7.4$ ) overnight, embedded in paraffin, sectioned and H\&E-stained for general histology. Lipid staining was performed by cryostat sectioning of the liver, followed by Oil Red O staining.

Quantitative PCR ( $q$ PCR) analysis. Total RNA was extracted from homogenised rat liver tissue with TRIzol reagent $(\sim 20 \mathrm{mg}$ of liver sample in $1 \mathrm{ml}$ TRIzol) and isolated by phenol/chloroform extraction. First-strand cDNA was synthesised from $5 \mu \mathrm{g}$ of total RNA using EasyScript First-Strand cDNA Synthesis SuperMix (Beijing Transgen Biotechnology Co., Ltd.,Beijing, China), according to the manufacturer's instructions. qPCR reactions were performed on an ABI 7300 instrument (Applied Biosystems Inc., Foster City, CA, USA), using Ultra SYBR Mixture [with 6-carboxy-x-rhodamine (ROX); Beijing CoWin Bioscience Co., Ltd., Beijing, China]. The total reaction volume was $20 \mu \mathrm{l}$ and contained $8 \mu \mathrm{l}$ of cDNA, $1 \mu \mathrm{l}$ of each primer and $10 \mu \mathrm{l}$ of $2 \mathrm{X}$ Ultra SYBR Mixture (with ROX). The amplification reactions were performed as follows: $10 \mathrm{~min}$ at $95^{\circ} \mathrm{C}, 40$ cycles of $95^{\circ} \mathrm{C}$ for $15 \mathrm{sec}, 60^{\circ} \mathrm{C}$ for $1 \mathrm{~min}$ and $72^{\circ} \mathrm{C}$ for $35 \mathrm{sec}$. GAPDH was used as an internal control and tests were performed in triplicate for each sample. The relative quantity was calculated using the $2^{-\Delta \Delta \mathrm{Ct}}$ method. The primers used for PPAR $\alpha$, CPT1A and GAPDH are listed in Table I.

Western blot analysis. Liver samples were homogenised in ice-cold RIPA lysis buffer with protease inhibitors of phenylmethanesulphonyl fluoride (PMSF) and incubated on an ice board for $30 \mathrm{~min}$. Lysates were clarified by centrifugation $(12,000 \mathrm{x} g$ for $10 \mathrm{~min})$ and the supernatants were collected and stored at $-80^{\circ} \mathrm{C}$. The protein concentrations were determined and equal amounts of protein $(40 \mu \mathrm{g})$ were subjected to electrophoresis on $10 \%$ sodium dodecyl sulphate polyacrylamide gels and then transferred to PVDF membranes using an electroblotting apparatus. Non-specific protein binding sites were blocked with PBS containing 0.1\% Tween-20 and $5 \%$ fat-free milk for $1 \mathrm{~h}$ at room temperature. The samples were then incubated overnight at $4^{\circ} \mathrm{C}$ with the following primary antibodies diluted in blocking buffer: PPAR $\alpha, \mathrm{CPT} 1 \mathrm{~A}, \mathrm{MTTP}$ and GAPDH. Subsequently, the membranes were washed three times and incubated for $2 \mathrm{~h}$ at room temperature with the appropriate HRP-conjugated secondary antibody in PBST (Beijing CoWin Bioscience Co., Ltd.). Immunoreactive bands were visualised using an enhanced chemiluminescence (ECL) detection system. The membranes were exposed to Kodak films (Carestream Health, Inc., Xiamen, China) for 5-10 min. Quantification of the resulting images was performed by densitometry with Gel-Pros Analyzer 4.0 software (Media Cybernetics, Inc., Bethesda, MD, USA) and the final readings were normalised against GAPDH.

Statistical analysis. The data are expressed as the mean \pm SEM. Statistical analysis was performed using one-way analysis of variance (ANOVA) with the SPSS 11.5 software package (SPSS, Inc., Chicago, IL, USA) to compare the experimental groups. $\mathrm{P}<0.05$ was considered to indicate a statistically significant difference.

\section{Results}

Body weight and VAT weight. The 12-week HFD caused a mild increase in body weight that was not statistically significant, and a significantly increased body weight gain $(\mathrm{P}<0.05)$ and VAT weight $(\mathrm{P}<0.01)$ in the HFD group compared with the SD group. Four weeks of OMT treatment led to a significantly lower body weight gain $(\mathrm{P}<0.01)$ and VAT weight $(\mathrm{P}<0.01)$ compared with the HFD group, and FF also reduced the body weight gain and VAT weight significantly $(\mathrm{P}<0.05$; Table II). During the study period, the calorie intake did not significantly differ amongst the groups, indicating that these effects occurred without being affected by calorie intake, and no adverse effects of the drugs, such as diarrhoea, were observed.

Biochemical parameters. Rats fed a HFD for 12 weeks demonstrated markedly increased serum TG, TC, FFA, ALT and liver TG levels $(\mathrm{P}<0.05$ or $\mathrm{P}<0.01)$ compared with the SD group. When compared with rats of the HFD group, OMT and FF treatment significantly lowered the serum TG, TC, FFA and liver TG levels $(\mathrm{P}<0.05)$. Moreover, OMT treatment significantly reduced the serum ALT level $(\mathrm{P}<0.01)$, which was 
Table II. Body weight, visceral adipose tissue weight and euglycemic clamping study of insulin sensitivity.

\begin{tabular}{|c|c|c|c|c|}
\hline Parameter & SD & HFD & OMT & $\mathrm{FF}$ \\
\hline Body weight prior to treatment (g) & $381.63 \pm 19.19$ & $354.87 \pm 23.73$ & $352.63 \pm 50.42$ & $356.12 \pm 38.63$ \\
\hline Body weight following treatment (g) & $397.00 \pm 26.58$ & $412.17 \pm 24.08$ & $362.17 \pm 39.96$ & $382.50 \pm 42.78$ \\
\hline Body weight gain (g) & $16.60 \pm 11.59$ & $58.10 \pm 16.60^{\mathrm{a}}$ & $10.11 \pm 8.09^{\mathrm{d}}$ & $26.10 \pm 12.64^{\mathrm{c}}$ \\
\hline VAT weight (g) & $11.60 \pm 0.70$ & $18.90 \pm 0.50^{\mathrm{b}}$ & $12.30 \pm 0.40^{\mathrm{d}}$ & $14.80 \pm 0.60^{\mathrm{c}}$ \\
\hline Caloric intake (kcal/rat/day) & $97.50 \pm 5.60$ & $105.30 \pm 7.30$ & $92.30 \pm 8.50$ & $93.60 \pm 6.90$ \\
\hline GIR (mg/kg/min) & $21.69 \pm 3.11$ & $14.72 \pm 2.13^{\mathrm{a}}$ & $19.55 \pm 3.29^{c}$ & $18.32 \pm 2.76^{\mathrm{c}}$ \\
\hline
\end{tabular}

Data are expressed as the mean \pm SEM. ${ }^{a} \mathrm{P}<0.05$, ${ }^{\mathrm{b}} \mathrm{P}<0.01$ vs. the $\mathrm{SD}$ group; ${ }^{\mathrm{c}} \mathrm{P}<0.05,{ }^{\mathrm{d}} \mathrm{P}<0.01$ vs. the HFD group. SD, standard diet; HFD, high-fructose diet; OMT, oxymatrine; FF, fenofibrate; VAT, visceral adipose tissue; GIR, glucose infusion rate.

Table III. Serum biochemical parameters and liver triglyceride (TG) contents.

\begin{tabular}{lcccc}
\hline Parameter & SD & HFD & OMT & FF \\
\hline Serum TG $(\mathrm{mmol} / \mathrm{l})$ & $0.44 \pm 0.26$ & $1.62 \pm 0.45^{\mathrm{b}}$ & $0.53 \pm 0.27^{\mathrm{d}}$ & $0.98 \pm 0.23^{\mathrm{c}}$ \\
Serum TC $(\mathrm{mmol} / \mathrm{l})$ & $1.01 \pm 0.21$ & $1.99 \pm 0.19^{\mathrm{a}}$ & $0.95 \pm 0.16^{\mathrm{d}}$ & $1.33 \pm 0.15^{\mathrm{c}}$ \\
Serum ALT $(\mathrm{IU} / \mathrm{l})$ & $29.41 \pm 7.89$ & $45.28 \pm 6.52^{\mathrm{a}}$ & $30.19 \pm 11.69^{\mathrm{c}}$ & $41.55 \pm 9.75$ \\
Serum FFA $(\mu \mathrm{mol} / \mathrm{l})$ & $669.92 \pm 142.68$ & $958.64 \pm 236.71^{\mathrm{b}}$ & $607.51 \pm 154.82^{\mathrm{d}}$ & $790.75 \pm 199.73^{\mathrm{c}}$ \\
FBG $(\mathrm{mmol} / \mathrm{l})$ & $5.56 \pm 0.21$ & $6.03 \pm 0.27$ & $5.90 \pm 0.32$ & $6.00 \pm 0.52$ \\
FinS $(\mathrm{ng} / \mathrm{ml})$ & $0.94 \pm 0.15$ & $1.83 \pm 0.31^{\mathrm{b}}$ & $1.15 \pm 0.29^{\mathrm{c}}$ & $1.23 \pm 0.32^{\mathrm{c}}$ \\
Liver TG contents $(\mathrm{mmol} / \mathrm{g})$ & $0.82 \pm 0.14$ & $3.13 \pm 0.33^{\mathrm{b}}$ & $0.90 \pm 0.15^{\mathrm{d}}$ & $2.07 \pm 0.28^{\mathrm{c}}$ \\
\hline
\end{tabular}

Data are expressed as the mean $\pm \mathrm{SEM} .{ }^{\mathrm{a}} \mathrm{P}<0.05,{ }^{\mathrm{b}} \mathrm{P}<0.01$ vs. the $\mathrm{SD}$ group; ${ }^{\mathrm{C}} \mathrm{P}<0.05,{ }^{\mathrm{d}} \mathrm{P}<0.01$ vs. the HFD group. SD, standard diet; HFD, high-fructose diet; OMT, oxymatrine; FF, fenofibrate; TG, triglyceride; TC, total cholesterol; ALT, alanine aminotransferase; FFA, free fatty acids; FBG, fasting blood glucose; FinS, fasting serum insulin.

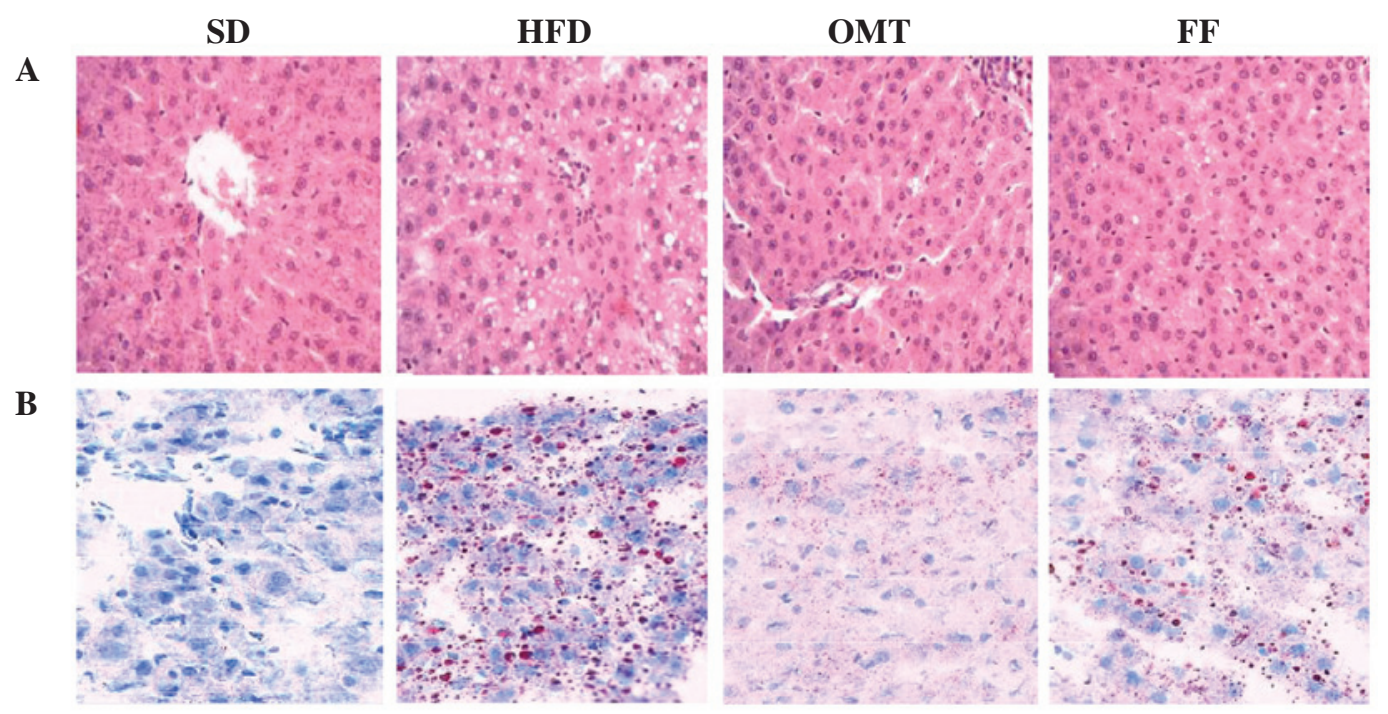

Figure 2. Typical photographs of liver histology from each group (magnification, x200). (A) Top panels, HE staining; (B) bottom panels, Oil Red O staining. $\mathrm{SD}$, standard diet; HFD, high fructose diet; OMT, oxymatrine; FF, fenofibrate.

elevated in the HFD group, whereas FF had only a slight effect on the ALT level (Table III).

Insulin sensitivity. The HFD increased the fasting serum insulin $($ FinS) level $(\mathrm{P}<0.01)$ and lowered the GIR $(\mathrm{P}<0.05)$ in the HFD group compared with SD group, whereas 4-week treatment with OMT or FF significantly decreased the FinS level and increased the GIR $(\mathrm{P}<0.05)$ compared with the HFD group. The fasting blood glucose (FBG) level was not altered by treatment with OMT or FF (Table II). 
A

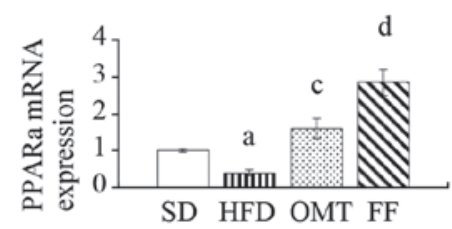

B

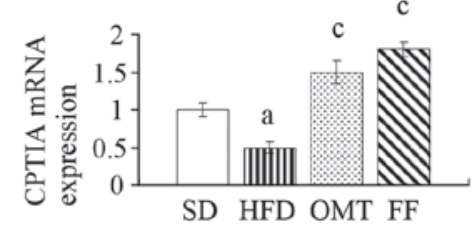

C

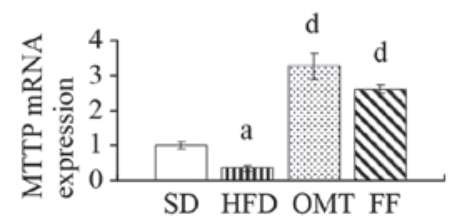

Figure 3. Effects of oxymatrine on (A) PPAR $\alpha$, (B) CPT1A and (C) MTTP mRNA expression in hepatic tissue following treatment for four weeks in NAFLD rats. Data are expressed as the mean \pm SEM. ${ }^{\mathrm{a}} \mathrm{P}<0.05,{ }^{\mathrm{b}} \mathrm{P}<0.01$ vs. the SD group; ${ }^{\mathrm{C}} \mathrm{P}<0.05,{ }^{\mathrm{d}} \mathrm{P}<0.01$ vs. the HFD group. $\mathrm{PPAR} \alpha$, peroxisome proliferator activated receptor- $\alpha$; CPT1A, carnitine palmitoyltransferase 1A; MTTP, microsomal triglyceride transfer protein; NAFLD, non-alcoholic fatty liver disease; SD, standard diet; HFD, high-fructose diet; OMT, oxymatrine; FF, fenofibrate.

A

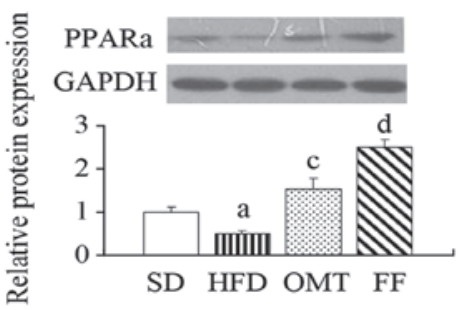

B

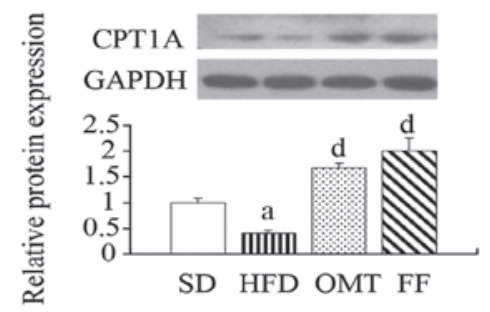

C

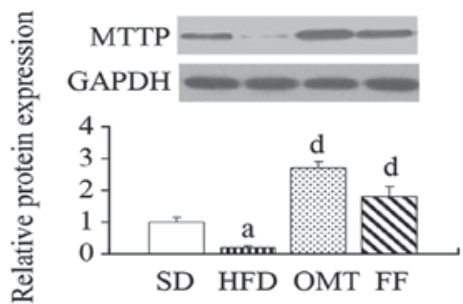

Figure 4. Effects of oxymatrine on (A) PPAR $\alpha$, (B) CPT1A and (C) MTTP protein expression in hepatic tissue after treatment for four weeks in NAFLD rats. Data are expressed as the mean \pm SEM. ${ }^{a} \mathrm{P}<0.05,{ }^{b} \mathrm{P}<0.01$ vs. the $\mathrm{SD}$ group; ${ }^{\mathrm{c}} \mathrm{P}<0.05,{ }^{\mathrm{d}} \mathrm{P}<0.01$ vs. the HFD group. PPAR $\alpha$, peroxisome proliferator activated receptor- $\alpha$; CPT1A, carnitine palmitoyltransferase 1A; MTTP, microsomal triglyceride transfer protein; NAFLD, non-alcoholic fatty liver disease; SD, standard diet; HFD, high-fructose diet; OMT, oxymatrine; FF, fenofibrate.
Histology examination. H\&E staining of liver sections from the HFD group revealed the derangement of liver cells and excessive vacuoles in the hepatocytes, particularly surrounding the central vein. These disorders were alleviated by treatment with OMT. Oil Red O staining confirmed that the vacuoles visible with H\&E staining were lipid droplets. Similar improvements were also observed in the FF group (Fig. 2).

qPCR and western blot analysis. The relative mRNA levels of PPAR $\alpha$, CPT1A and MTTP were downregulated in the livers of HFD-fed rats compared with the SD group, whereas OMT or FF treatment upregulated the expression of PPAR $\alpha$, CPT1A and MTTP (P<0.05; Fig. 3). Consistent with alterations in the mRNA expression levels, western blot analysis of liver protein extracts verified corresponding changes in the protein levels of PPAR $\alpha$, CPT1A and MTTP $(\mathrm{P}<0.05$; Fig. 4).

\section{Discussion}

The present study revealed that OMT improves the biochemical and histological signatures of HFD-induced NAFLD rats with problems such as hypertriglyceridaemia, IR and liver steatosis. This result is consistent with a recent study by Zeng et al (14), which demonstrated that oral administration of OMT for one week eliminated hepatic steatosis in fatty liver mice. We further explored the mechanisms underlying the reduction in liver fat accumulation.

As mentioned previously, hepatic steatosis is the hallmark of NAFLD, and is also the product of lipid metabolism imbalance. From this perspective, two possible mechanisms responsible for the attenuation of NAFLD pathology in the OMT treatment group may be considered: one is a decrease in the FFA influx to the liver and the second is an increase in the lipid outflux through the promotion of lipid discharge from the liver and the $\beta$-oxidation of lipids in the liver.

With regard to the first pathway, it has been demonstrated that $\sim 60 \%$ of TGs deposited in the liver in NAFLD originate from circulating non-esterified fatty acids (21). Currently, adipose tissue is considered to be the main source of FFA and visceral fat is more prone to lipolysis than subcutaneous fat. Furthermore, impaired insulin action may increase lipolysis and reduce the utilisation of FFA in adipose tissues (4). Therefore, reducing body weight, specifically visceral fat, and improving insulin sensitivity may be effective strategies for decreasing FFA influx to the liver.

In this study, the HFD rats exhibited increased VAT weights, marked IR and higher serum FFA levels compared with the SD group, which signifies an enhanced influx of FFA to hepatocytes. This increases hepatic TG production and lipid availability. However, OMT decreased body weight gain and VAT weight, elevated the GIR and reduced serum FFA levels, indicating a decreased FFA influx to hepatocytes.

With regard to the second pathway, we examined the PPAR $\alpha$, CPT1A and MTTP gene and protein expression levels, which are important for the evaluation of FFA $\beta$-oxidation and the outflow of lipids from the liver. PPAR $\alpha$ belongs to a superfamily of nuclear, ligand-activated transcription factors. PPAR $\alpha$ is important in the catabolism of fatty acids and is involved in the pathogenesis and treatment of NAFLD (22). 
Increasing evidence has suggested that PPAR $\alpha$ activation normalises serum TG and FFA levels, improves liver insulin sensitivity (23) and prevents lipid accumulation in the liver (24) by regulating the expression of pivotal enzymes, including CPT1A and MTTP. CPT1A is the key regulatory enzyme of mitochondrial fatty acid oxidation and MTTP is the essential enzyme of VLDL assembly and export from the liver.

In this study, the mRNA and protein levels of PPAR $\alpha$ and CPT1A were decreased in the HFD group compared with the SD group $(\mathrm{P}<0.01)$, indicative of the impaired $\beta$-oxidation of FFA, which may affect the imbalance of lipid metabolism toward lipid accumulation. Similar changes were previously reported in patients with NAFLD (25). In the treatment groups, either OMT or FF administration for four weeks significantly increased PPAR $\alpha$ and CPT1A expression. A previous study demonstrated that the overexpression of CPT1A was accompanied by a $69 \%$ reduction in hepatic TG accumulation in obese Sprague-Dawley rats (26). In this study, we observed a similar change in CPT1A expression and a $71 \%$ reduction in liver TG contents in the OMT treatment group, consistent with the previous study.

MTTP, discovered by Wetterau et al (27) in 1984 , is necessary for the assembly and secretion of apoB100-containing lipoproteins (e.g., VLDL and LDL) to export lipids from the liver. The downregulation of MTTP gene expression in ob/ob mice facilitates intracellular fat accumulation in hepatocytes, which increases susceptibility to hepatic steatosis (28). However, the overexpression of MTTP decreases fat accumulation and increases the secretion of apoB100-containing particles (29). In this study, the mRNA and protein expression levels of MTTP in the rat liver decreased in the HFD group compared with the SD group. After four weeks of treatment with OMT, MTTP expression was upregulated. In the FF group, the MTTP mRNA and protein levels were increased. These results suggested that treatment with OMT or FF increases liver TG export through the activation of liver MTTP and PPAR $\alpha$, thereby reducing the hepatic TG accumulation and markedly attenuating the pathology of NAFLD.

In this study, OMT and FF treatment decreased the serum TG, TC and liver TG levels compared with the HFD group, but the administration of OMT decreased the levels more efficiently than FF treatment (-52 vs. $-33 \%$ for serum TC, -67 vs. $-40 \%$ for serum TG and -71 vs. $-34 \%$ for liver TG, respectively). Furthermore, OMT reversed elevated ALT levels in the fatty liver, whereas FF displayed only a mild decrease, which suggests that OMT is more effective than FF for the modulation of liver function. Although these results indicate that treatment with OMT is more advantageous than with FF, their underlying mechanisms remain unknown.

A limitation of this study was that several details with regard to lipid metabolism require clarification following treatment with OMT, such as fatty acid absorption, fatty acid uptake and fatty acid synthesis. Further studies are required to evaluate the change and degree of the complex transcriptional network in this process.

In conclusion, this study demonstrated that OMT had a notable beneficial effect and may be useful in the treatment of hepatic steatosis associated with visceral obesity, dyslipidaemia and IR. The underlying mechanism was shown to be associated with the modulation of abnormal lipid metabolism and reversed lipid imbalance in the liver. Moreover, one molecular mechanism of OMT treatment lies in its effect on PPAR $\alpha$ activation and the corresponding upregulation of PPAR $\alpha$ target enzymes, including CPT1A and MTTP. The present data indicate that these findings should be extended to clinical trials in order to demonstrate the effectiveness of OMT treatment in NAFLD.

\section{Acknowledgements}

This work was supported in partby grants from the International Cooperation Project of Hebei province Department of Science and Technology (11396406-D).

\section{References}

1. Tiniakos DG, Vos MB and Brunt EM: Nonalcoholic fatty liver disease: pathology and pathogenesis. Annu Rev Pathol 5: 145-171, 2010.

2. Sheng L, Cho KW, Zhou Y, et al: Lipocalin 13 protein protects against hepatic steatosis by both inhibiting lipogenesis and stimulating fatty acid $\beta$-oxidation. J Biol Chem 286: 38128-38135, 2011.

3. Utzschneider KM and Kahn SE: Review: The role of insulin resistance in nonalcoholic fatty liver disease. J Clin Endocrinol Metab 91: 4753-4761, 2006.

4. Fabbrini E, Mohammed BS, Magkos F, Korenblat KM, Patterson BW and Klein S: Alterations in adipose tissue and hepatic lipid kinetics in obese men and women with nonalcoholic fatty liver disease. Gastroenterology 134: 424-431, 2008.

5. Smith BW and Adams LA: Non-alcoholic fatty liver disease. Crit Rev Clin Lab Sci 48: 97-113, 2011.

6. Gaemers IC, Stallen JM, Kunne C, et al: Lipotoxicity and steatohepatitis in an overfed mouse model for non-alcoholic fatty liver disease. Biochim Biophys Acta 1812: 447-458, 2011.

7. Kersten S, Desvergne B and Wahli W: Roles of PPARs in health and disease. Nature 405: 421-424, 2000.

8. Améen C, Edvardsson U, Ljungberg A, et al: Activation of peroxisome proliferator-activated receptor alpha increases the expression and activity of microsomal triglyceride transfer protein in the liver. J Biol Chem 280: 1224-1229, 2005.

9. Ye JM, Doyle PJ, Iglesias MA, Watson DG, Cooney GJ and Kraegen EW: Peroxisome proliferator-activated receptor (PPAR)-alpha activation lowers muscle lipids and improves insulin sensitivity in high fat-fed rats: comparison with PPAR-gamma activation. Diabetes 50: 411-417, 2001.

10. Seo YS, Kim JH, Jo NY, et al: PPAR agonists treatment is effective in a nonalcoholic fatty liver disease animal model by modulating fatty-acid metabolic enzymes. J Gastroenterol Hepatol 23: 102-109, 2008.

11. Cui X, Wang Y, Kokudo N, Fang D and Tang W: Traditional Chinese medicine and related active compounds against hepatitis B virus infection. Biosci Trends 4: 39-47, 2010.

12. Cao YG, Jing S, Li L, et al: Antiarrhythmic effects and ionic mechanisms of oxymatrine from Sophora flavescens. Phytother Res 24: 1844-1849, 2010.

13. Deng ZY, Li J, Jin Y, Chen XL and Lü XW: Effect of oxymatrine on the p38 mitogen-activated protein kinases signalling pathway in rats with $\mathrm{CCl} 4$ induced hepatic fibrosis. Chin Med J (Engl) 122: 1449-1454, 2009

14. Zeng XY, Zhou X, Xu J, et al: Screening for the efficacy on lipid accumulation in 3T3-L1 cells is an effective tool for the identification of new anti-diabetic compounds. Biochem Pharmacol 84: 830-837, 2012.

15. Yuan LJ, Lu X, Wang J, Zheng TZ, Qu SY and Zhang XY: Effects of matrine on weight, serum lipids and anti-oxidative capacity in high-fatted rats. Lishizhen Medicine and Materia Medica Research 9: 2062-2064, 2008.

16. Zhang HF, Shi LJ, Song GY et al: Protective effects of matrine against progression of high-fructose diet-induced steatohepatitis by enhancing antioxidant and anti-inflammatory defences involving Nrf2 translocation. Food Chem Toxicol 55: 70-77, 2013. 
17. Xing Y, Yan F, Liu Y, Liu Y and Zhao Y: Matrine inhibits 3T3-L1 preadipocyte differentiation associated with suppression of ERK1/2 phosphorylation. Biochem Biophys Res Commun 396: 691-695, 2010

18. Thorburn AW, Storlien LH, Jenkins AB, Khouri S and Kraegen EW: Fructose-induced in vivo insulin resistance and elevated plasma triglyceride levels in rats. Am J Clin Nutr 49: $1155-1163,1989$.

19. Ren LP, Chan SM, Zeng XY, et al: Differing endoplasmic reticulum stress response to excess lipogenesis versus lipid oversupply in relation to hepatic steatosis and insulin resistance. PLoS One 7: e30816, 2012.

20. Kraegen EW, James DE, Bennett SP and Chisholm DJ: In vivo insulin sensitivity in the rat determined by euglycemic clamp. Am J Physiol 245: E1-E7, 1983.

21. Donnelly KL, Smith CI, Schwarzenberg SJ, Jessurun J, Boldt MD and Parks EJ: Sources of fatty acids stored in liver and secreted via lipoproteins in patients with nonalcoholic fatty liver disease. J Clin Invest 115: 1343-1351, 2005.

22. Postic C and Girard J: Contribution of de novo fatty acid synthesis to hepatic steatosis and insulin resistance: lessons from genetically engineered mice. J Clin Invest 118: 829-838, 2008.

23. Chou CJ, Haluzik M, Gregory C, et al: WY14, 643, a peroxisome proliferator-activated receptor alpha (PPARalpha) agonist, improves hepatic and muscle steatosis and reverses insulin resistance in lipoatrophic A-ZIP/F-1 mice. J Biol Chem 277: 24484-24489, 2002.
24. Ip E, Farrell GC, Robertson G, Hall P, Kirsch R and Leclercq I: Central role of PPARalpha-dependent hepatic lipid turnover in dietary steatohepatitis in mice. Hepatology 38: 123-132, 2003.

25. Kohjima M, Enjoji M, Higuchi N, et al: Re-evaluation of fatty acid metabolism-related gene expression in nonalcoholic fatty liver disease. Int J Mol Med 20: 351-358, 2007.

26. Stefanovic-Racic M, Perdomo G, Mantell BS, Sipula IJ, Brown NF and O'Doherty RM: A moderate increase in carnitine palmitoyltransferase 1a activity is sufficient to substantially reduce hepatic triglyceride levels. Am J Physiol Endocrinol Metab 294: E969-E977, 2008.

27. Wetterau JR and Zilversmit DB: A triglyceride and cholesteryl ester transfer protein associated with liver microsomes, J Biol Chem 259: 10863-10866, 1984.

28. Stefano JT, de Oliveira CP, Corrêa-Giannella ML, et al: Nonalcoholic steatohepatitis (NASH) in ob/ob mice treated with yo jyo hen shi ko (YHK): effects on peroxisome proliferator-activated receptors (PPARs) and microsomal triglyceride transfer protein (MTP). Dig Dis Sci 52: 3448-3454, 2007.

29. Chen Z, Newberry EP, Norris JY, et al: ApoB100 is required for increased VLDL-triglyceride secretion by microsomal triglyceride transfer protein in ob/ob mice. J Lipid Res 49: 2013-2022, 2008. 